\title{
Factors Associated with Vitamin D Deficiency Among Adolescents in the Korea
}

\author{
Ji Won Oak \\ Dept. of Nursing, Tongmyong University \\ jiwonoak@gmail.com
}

\begin{abstract}
The purpose of this research is to understand the factors related to the vitamin $D$ deficiency. This research secondarily analyzed the raw data of 'the $6^{\text {th }}$ Korean National Health and Nutrition Examination Surveys (2013-2014)', which had been investigated by the Korea Centers for Disease Control and Prevention. There had been a total of 561 subjects. The data were analyzed with the $t$-test, the $\chi^{2}$-test, and the logistic regression analysis. The results of the research were the regarding the vitamin $D$ deficiency of the youths, the prevalence rate was higher the older the age $(p=.041)$. And in the case of having engaged in the walking exercises $(p=.011)$, the prevalence rate was low. In this research, it was able to confirm that the age and the walking exercise are the related factors of the vitamin $D$ deficiency of the youths. As a result, in order to alleviate the vitamin D deficiency of the junior high school students, the nursing intervention and the development of the educational program that consider the above variables and the verification of their effects is demanded.
\end{abstract}

Keywords: Adolescent, Vitamin D, Deficiency, Health, Promotion, Behavior

\section{Introduction}

It has been known that vitamin $\mathrm{D}$ is indispensable to the growth and the maintenance of the physique and the maintenance of the homeostasis of calcium. Recently, there have been the reports of the results of the researches that they are involved with regard to not only the musculoskeletal system but, also, the malignant tumors [1][2] of the breast cancer, the large intestine cancer, the prostate cancer, etc., diabetes [3], high blood pressure [4], cardiovascular disorders [5], the contagious diseases, the immunity system diseases, etc. [1]. As such, the roles of vitamin $\mathrm{D}$ have been newly illuminated.

In Korea, too, there has been the interest in vitamin D. It was selected as a physical examination item of the $6^{\text {th }}$ Korean National Health and Nutrition Examination Surveys in 2007. The large-scale investigations have been proceeded with. The reports and the researches on the insufficiency of vitamin D and the condition of the deficiency of vitamin D among Koreans began [6]. Based on the results of the $6^{\text {th }}$ Korean National Health and Nutrition Examination Surveys in 2008, Choi et al [3] pointed out, especially, the problem that the insufficiency and the deficiency of vitamin D is very serious among the young generation while saying that the insufficiency of vitamin $\mathrm{D}$ is a very frequent problem among the Koreans. Because, the $1^{\text {st }}$ Korean National Health and Nutrition Examination Surveys in the $5^{\text {th }}$ term in 2010, it was reported [7] that $78 \%$ of the children and the youths of Korea have

Article history:

Received (July 31, 2016), Review Result (September 16, 2016), Accepted (October 23, 2016) 
been showing the deficiency of vitamin $\mathrm{D}(<20 \mathrm{ng} / \mathrm{dl})$, we can find out that the interest in the deficiency of vitamin $\mathrm{D}$ during the adolescent period has been demanded.

Among the researches on the deficiency of vitamin D that have been reported in Korea until now, especially, the researches with the youths whose health behaviors are formed as the subjects are in the condition of being meager. Among the phases of the development, the adolescent period is an important time during which the proper and healthy life habits are formed and set in. When taking into account the fact that the proper health behaviors formed during the adolescent period are the important factors in the management of vitamin $\mathrm{D}$, which has been illuminated as a factor of the chronic diseases, it is thought that the accurate confirmation of the related factors must take place first together with the investigations on the prevalence rate of the deficiency of vitamin D among the youths.

As a result, in this research, by analyzing the factors related to this, it is intended to provide the basic data and the materials for the development of an effective nursing intervention program for the promotion of the health of the youths.

\section{Methods}

\subsection{Study design}

This research is a cross-sectional, descriptive research for understanding the factors that influence this by using the raw data of the $6^{\text {th }}$ Korean National Health and Nutrition Examination Surveys (2013-2014) of the Korea Centers for Disease Control and Prevention.

\subsection{Samples and setting}

This research secondarily analyzed the raw data of the $6^{\text {th }}$ Korean National Health and Nutrition Examination Surveys in the $1^{\text {st }}$ and $2^{\text {nd }}$ years $(2013-2014)$ of the $6^{\text {th }}$ term as announced on March 31, 2016. Regarding the samples of the $6^{\text {th }}$ Korean National Health and Nutrition Examination Surveys of the $6^{\text {th }}$ term, which are the raw data, the data of the population and housing census were made into the frames. The frames were stratified based on the cities, provinces, dongs, eups, myeons, and the types of the housings (ordinary houses and apartments). And the 2-phase, stratified, cluster sample extraction method (two-stage cluster sampling), which uses the ratios of the residential areas, the ratio between the head of the family and the academic background, and the others of the like as the intrinsic stratification standards, was applied [8].

Regarding the investigation areas, 576 areas were extracted for 3 years, for 6 terms (20142015), and with 192 areas per year. 20 sample households were selected by using the systematic sampling method among the appropriate households excluding the facilities, including the homes for the aged, the military, the prisons, etc., the foreigner households, etc. from within the sample investigation areas. Within the sample households, all the members of the households aged 1 year or older who satisfy the requirements for being a reasonable member of the household were selected. And, in 2013, 8,018 persons were investigated. And, in 2014, 7,550 persons were investigated.

After selecting 1,272 youths, who are 12 years old or older and who are 18 years old or younger, who fit the purpose of this research among the total of the 15,568 people who are the samples of the raw data, the 561 persons regarding which the vitamin D inspection was carried out were selected as the final subjects. 


\subsection{Instruments}

Among the raw data of the $6^{\text {th }}$ Korean National Health and Nutrition Examination Surveys, the measurement tools were organized with the 21 questions on the general, special characteristics, the 34 questions on the special characteristics related to the health behaviors, the questionnaire with a total of 55 questions, and the inspection results regarding the 20 items of the physical examination index and the 16 items of the nutrition index.

\subsection{Data analyses}

The data that were collected were analyzed by using the SPSS WIN 23.0 program.

In order to understand the risk factors that have the influences on the vitamin D deficiency of the youths, a logistic regression analysis was carried out.

\section{Results}

\subsection{The prevalence rate of the youths' vitamin $D$ deficiency}

In this research, the group with the vitamin D deficiency was 427 persons (76.1\%).

Table 1. The prevalence rate of the youths' vitamin D deficiency $(\mathrm{N}=561)$

\begin{tabular}{|c|c|c|c|c|}
\hline \multicolumn{2}{|c|}{ Variables } & $\begin{array}{c}\text { Standard value } \\
(\mathrm{ng} / \mathrm{mL})\end{array}$ & $\mathrm{n}(\%)$ & $\mathrm{n}(\%)$ \\
\hline \multirow{2}{*}{$\begin{array}{c}\text { Vitamin D } \\
\text { deficiency group }\end{array}$} & Severe Deficiency & $<10$ & $67(11.9)$ & \multirow{2}{*}{$427(76.1)$} \\
\cline { 2 - 4 } & Deficiency & $10-19.9$ & $360(64.2)$ & \multirow{2}{*}{$134(23.9)$} \\
\hline \multirow{2}{*}{$\begin{array}{c}\text { Normal } \\
\text { group }\end{array}$} & Insufficiency & $20-29.9$ & $124(22.1)$ & \\
\cline { 2 - 4 } & Sufficiency & $\geq 30$ & $10(1.8)$ & \\
\hline
\end{tabular}

\subsection{The factors that have the influences on the vitamin $D$ deficiency of the youths}

The risk factors that have the influences on the prevalence rate of the vitamin D deficiency of the youths were verified with a logistic regression analysis by having the variables that appeared significant with regard to the general, special characteristics, the health-related special characteristics, the physical examination index, and the nutrition index as the independent variables. It this research, it appeared that the -2LL (-2 log likelihood) value of the logistic regression model was 489.865 , the Nagelkerke's $\mathrm{R}^{2}$ was 0.168 , the chi-square was 59.035 ( $\mathrm{p}<.001)$, and the degree of the accuracy of the prediction was $77.6 \%$. And the related factors that have the influences on the prevalence rate of the deficiency of vitamin D among the youths appeared to be the age and whether or not the walking exercise is engaged in.

Specifically, the more the age increases by 1 year at a time, the prevalence rate of the vitamin D deficiency gets higher by 1.150 times. And it appeared that, the more one engages in the walking exercise, the prevalence of the vitamin D deficiency was 0.229 times lower than the case in which the walking exercise was not engaged in [Table 2].

\section{Discussion and conclusions}

This research was a research that was attempted in order to understand the factors related to the vitamin D deficiency among the youths in. The raw data of the Korean National Health and Nutrition Examination Surveys in the $1^{\text {st }}$ and $2^{\text {nd }}$ years $(2013-2014)$ of the $6^{\text {th }}$ term that 
were announced on March 31, 2016 were secondarily analyzed. The prevalence rate of the vitamin D deficiency of the youths in Korea was $76.1 \%$. And, as the related factors, the age and whether or not one has engaged in the walking exercise were confirmed.

Table 2. The factors that have the influences on the vitamin D deficiency of the youths $(\mathrm{N}=561)$

\begin{tabular}{|c|c|c|c|c|c|c|c|}
\hline & & & & & & \multicolumn{2}{|c|}{$95 \% \mathrm{CI}^{*}$} \\
\hline Variable & $\beta$ & S.E. & Wald & $\mathrm{p}$ & $\begin{array}{c}\text { Odds } \\
\text { ratio }\end{array}$ & Lower & Upper \\
\hline Age & .140 & .068 & 4.156 & .041 & 1.150 & 1.005 & 1.315 \\
\hline $\begin{array}{c}\text { Whether or not one has } \\
\text { engaged in the walking } \\
\text { exercise. }\end{array}$ & -1.473 & .580 & 6.460 & .011 & .229 & .074 & .714 \\
\hline
\end{tabular}

*CI=confidence interval

The prevalence rate of the vitamin D deficiency among the youths (12-14 years old) in Korea that was confirmed in this research was $76.1 \%$. This is a result that is similar to the research by Jung [9] which reported that the prevalence rate of the vitamin D deficiency among 2,193 youths in the $5^{\text {th }}$ term Korean National Health and Nutrition Examination Surveys was $81.5 \%$. We can find out that the vitamin D deficiency among the Korean youths is on a serious level. Due to the lowering of the physical activities resulting from the increase of the age, with the exposure to the sun's rays getting reduced, although it was predicted that the amount of the vitamin D created in the skin will be reduced, in the researches by Lappe [10] and Kim and Kang [11], it was reported that, the more the age increases, the level of the vitamin D increases and that the vitamin D deficiency is the smallest among the males in their 60 's and the females in their 50's and, in contrast, there is a lot of the deficiency among the young bracket. Because there have not been any preceding researches that analyzed the factors that influence the levels of vitamin D with the youths as the subjects, a direct comparison is difficult. However, it is thought that this is related to the increase of the use of the computers and the mobile phones and the decrease of the outdoor activities on the part of the youths due to the educational environment that is centered on the entrance examination.

Also, in this research, the vitamin D deficiency among the youths had the differences according to whether or not one has engaged in the walking exercise in the recent 1 month. And it was confirmed that whether or not one has engaged in the walking exercise is a relevant factor with regard to the vitamin D deficiency. Scragg and Camargo [12] reported that, regarding the physical activities, there is a correlation between the level of vitamin D in the blood and the independent amount with no relationship to the race, age, gender, and season. Thereby, it explains the results of this research.

Based on the results of this research, I intend to make the following proposals. Firstly, the nursing interest in the vitamin D deficiency of the youths must be increased. And a plan for efficiently managing the vitamin D deficiency must be prepared by tracing and comparing the prevalence rate of the vitamin D deficiency among the youths and the related factors by expanding the variables, at the same time. Secondly, this research has the strong point that it understood the prevalence rate of the vitamin D deficiency among the youths in Korea and the related factors by analyzing the large-scale data from nationwide by using the raw data of 
the $6^{\text {th }}$ Korean National Health and Nutrition Examination Surveys (2013-2014) of the Korea Centers for Disease Control and Prevention. However, as there is the limitation of not having been able to consider all the detailed variables as the factors related to the vitamin $\mathrm{D}$ deficiency, the detailed analyses of the general, special characteristics, the health-related, special characteristics, the physical examination index, etc. and the establishment of a comprehensive model are demanded for the future.

\section{References}

[1] M. F. Holick, N. C. Binkley, H. A. Bischoff-Ferrari, C. M. Gordon, D. A. Hanley, R. P. Heaney, M. H. Murad, and C. M. Weaver: "Evaluation, treatment, and prevention of vitamin d deficiency: An endocrine society clinical practice guideline," The Journal of Clinical Endocrinology \& Metabolism, vol.96, no.7, pp.1911-1930, (2011) DOI:10.1210/jc.2011-0385

[2] E. Giovannucci, "The epidemiology of vitamin d and cancer incidence and mortality: A Review (United States)," Cancer Causes and Control, vol.16, no.2, pp.83-95, (2005) DOI:10.1007/s10552-004-1661-4

[3] H. S. Choi, K. Kim, C. Lim, S. Y. Rhee, Y. Hwang, K. M. Kim, K. J. Kim, Y. Rhee, and S. Lim, "Low serum vitamin $\mathrm{d}$ is associated with high risk of diabetes in korean adults," The Journal of Nutrition, vol.141, no.8, pp.1524-1528, (2011) DOI:10.3945/jn.111.139121

[4] J. P. Forman, E. Giovannucci, M. D. Holmes, H. A. Bischoff-Ferrari, S. S. Tworoger, W. C. Willett, and G. C. Curhan, "Plasma 25-Hydroxyvitamin D levels and risk of incident hypertension," Hypertension, vol. 49, pp.1063-1069, (2007)

[5] J. H. Lee and J. H. O'Keefe, D. Bell, D. D. Hensrud, and M. F. Holick, "Vitamin D deficiency: An Important, common, and easily treatable cardiovascular risk factor?" Journal of the American College of Cardiology, vol.52, no.24, pp.1949-1956, (2008) DOI:10.1016/j.jacc.2008.08.050

[6] M. Kim, W. Na, and C. Sohn, "Correlation between Vitamin D and cardiovascular disease predictors in overweight and obese Koreans," Journal of Clinical Biochemistry and Nutrition, vol.52, no.2, pp.167-171, (2013) DOI:10.3164/jcbn.12-81

[7] S. Kwon, "Factors Associated with Vitamin D deficiency in Korea: Using the Korea national health and nutrition examination survey," M.S. Thesis, Korea University, (2013)

[8] Korea Centers for Disease Control and Prevention, "Using guidelines for the Korea youth health risk behavior web-based survey (KYHRBWS) raw data," Seoul, Author, (2014)

[9] M. Y. Jung, "Relationship of vitamin D level, physical activity and walking with mental health of youth: Korean National Health and Nutrition Examination Survey 2010-2012,” M.S. Thesis, Catholic University, (2015)

[10] J. M. Lappe, "The role of Vitamin D in human health: A paradigm shift," Journal of Evidence-Based Complementary \& Alternative MedicineJ, vol.16, no.1, pp.58-72, (2011) DOI:10.1177/1533210110392952

[11] J. I. Kim and M. J. Kang, "Recent consumption and physiological status of Vitamin D in Korean population," Food Industry and Nutrition, vol.17, no.2, pp.7-10, (2012)

[12] R. Scragg and C. A. Jr. Camargo, "Frequency of leisure-time physical activity and serum 25Hydroxyvitamin D levels in the us population: Results from the third national health and nutrition examination survey," American Journal of Epidemiology, vol.168, no.6, pp.571-586, (2008) DOI:10.1093/aje/kwn163 
Factors Associated with Vitamin D Deficiency among Adolescents in the Korea

This page is empty by intention. 